\title{
Abnormal Stress Responsivity in a Rodent Developmental Disruption Model of Schizophrenia
}

\author{
Eric C Zimmerman*,', Mark Bellaire', Samuel G Ewing ${ }^{1,2}$ and Anthony A Grace' \\ 'Departments of Neuroscience, Psychiatry and Psychology, University of Pittsburgh, Pittsburgh, PA, USA
}

\begin{abstract}
Although numerous studies have implicated stress in the pathophysiology of schizophrenia, less is known about how the effects of stress interact with genetic, developmental, and/or environmental determinants to promote disease progression. In particular, it has been proposed that in humans, stress exposure in adolescence could combine with a predisposition towards increased stress sensitivity, leading to prodromal symptoms and eventually psychosis. However, the neurobiological substrates for this interaction are not fully characterized. Previous work in our lab has demonstrated that rats born to dams administered with the DNA-methylating agent methylazoxymethanol acetate (MAM) at gestational day 17 exhibit as adults behavioral and anatomical abnormalities consistent with those observed in patients with schizophrenia. Here, we examined behavioral and neuroendocrine responses to stress in the MAM model of schizophrenia. MAM-treated male rats were exposed to acute and repeated footshock stress at prepubertal, peripubteral, and adult ages. Ultrasonic vocalizations (USVs), freezing, and corticosterone responses were quantified. We found that juvenile MAM-treated rats emitted significantly more calls, spent more time vocalizing, emitted calls at a higher rate, and showed more freezing in response to acute footshock stress when compared with their saline (SAL) treated counterparts, and that this difference is not present in older animals. In addition, adolescent MAM-treated animals displayed a blunted HPA axis corticosterone response to acute footshock that did not adapt after 10 days of stress exposure. These data demonstrate abnormal stress responsivity in the MAM model of schizophrenia and suggest that these animals are more sensitive to the effects of stress in youth.

Neuropsychopharmacology (2013) 38, 2131 -2139; doi:10.1038/npp.2013.1 I0; published online 5 June 2013
\end{abstract}

Keywords: schizophrenia/antipsychotics; behavioral science; animal models; psychiatry and behavioral sciences; MAM model

\section{INTRODUCTION}

Stress is believed to contribute to the pathogenesis of a variety of psychiatric illnesses (Belujon and Grace, 2011; Roozendaal et al, 2009; Touma, 2011). In particular, evidence suggests that early life stress is an important factor in the etiology of schizophrenia, a developmental disorder that typically manifests in adolescence or early adulthood. Stressful life events can precipitate or exacerbate the psychotic symptoms of schizophrenia (Corcoran et al, 2003; Meyer-Lindenberg and Tost, 2012) and psychosocial stressors increase the risk for developing the disease (Lim and Chong, 2009). It has been suggested that individuals who are at risk for schizophrenia are more susceptible to the effects of stress and that the interaction between a genetic or developmental predisposition and stress in early

\footnotetext{
*Correspondence: EC Zimmerman, Departments of Neuroscience, Psychiatry and Psychology, University of Pittsburgh School of Medicine, Medical Scientist Training Program, 526 Scaife Hall, 3550 Terrace Street, Pittsburgh, PA 1526I, USA, Tel: +I 262893 8186, Fax: + 4126249198, E-mail: ecz5@pitt.edu

${ }^{2}$ Current Address: Experimentelle Psychiatrie-Klinik für Psychiatrie und Psychotherapie, Universitätsklinik Carl Gustav Carus an der Technischen Universität Dresden, Fetscherstrasse 74, D-0 307 Dresden, Germany.

Received I0 January 20 I3; revised I May 20 I3; accepted 3 May 20 I3; accepted article preview online 8 May 2013
}

life could promote symptom onset (Benes, 1997; Tsuang, 2000; Walker et al, 2008). Indeed, in children at risk for schizophrenia, those that show abnormally high responses to stress tend to be those that convert to schizophrenia (Johnstone et al, 2002; Owens et al, 2005). However, the neurobiological substrate for this susceptibility remains unclear.

In order to address this question, we examined behavioral and neuroendocrine responses to stress in a well-validated animal model of schizophrenia. Rats born to dams administered with the mitotoxin methylazoxymethanol acetate (MAM; Nagata and Matsumoto, 1969) on gestational day (GD) 17 display multiple neural and behavioral abnormalities that mirror those seen in schizophrenia, including thinning of limbic cortices, deficits in rhythmic activity in frontal cortex, decreased prepulse inhibition of the startle reflex, and deficits in latent inhibition, among others (Flagstad et al, 2004; Grace and Moore, 1998; Le Pen et al, 2006; Lodge and Grace, 2008; Moore et al, 2006). Importantly, previous work from our laboratory suggests that adult MAM animals are more susceptible to alterations in synaptic plasticity associated with stress (Belujon et al, 2013; Goto and Grace, 2006).

In this study, we examined stress responsivity in MAMtreated animals at prepubertal, peripubertal, and adult ages using two objective and quantifiable measures: USVs and HPA axis activation. USVs are closely linked to affective states in rats (Kim et al, 2010; Portfors, 2007; Schwarting and 
Wöhr, 2012). Rats exposed to footshock, airpuff startle, and intermittent cold-water swim stress, as well as alcohol or heroin withdrawal, emit robust $22 \mathrm{kHz}$ vocalizations (Brudzynski, 2001; Williams et al, 2012). In addition, USVs have been shown to predict stress resilience in rats challenged with intermittent cold-water swim stress (Drugan et al, 2009). The HPA axis serves to maintain physiological homeostasis in response to stress, a function that is robustly influenced by regulatory input from the limbic cortices (Jankord and Herman, 2008; McEwen and Gianaros, 2010). Numerous studies have highlighted limbic system abnormalities in patients diagnosed with schizophrenia and in animal models of the disease (Chance et al, 2002; Grace, 2010; Lisman et al, 2010). In addition, patients with schizophrenia show abnormal HPA axis responses to stressors (Walker et al, 2008, 2004).

Given these findings, we examined USV and HPA axis responses to acute and repeated stress in the MAM model of schizophrenia. We hypothesized that juvenile and adolsecent MAM-treated animals would exhibit altered physiological responses to both acute and repeated stressors as compared with their SAL counterparts. Single cohorts of MAM and SAL rats were exposed to inescapable footshock stress throughout development at prepubertal, peripubertal, and adult time points. In these animals, we recorded and quantified USVs emitted in response to acute footshock and analyzed total number of calls, call duration, and call frequency, as well as freezing behavior exhibited during recording sessions. In a separate experiment, we measured plasma corticosterone levels following acute as well as repeated footshock in peripubertal MAM and SAL rats. Together, our findings suggest that USV responses to stress are exaggerated and the neuroendocrine stress response is abnormal in the MAM model of schizophrenia.

\section{METHODS}

\section{Animals and MAM Treatment}

All experiments were performed in accordance with the guidelines outlined in the United States Public Health Service Guide for the Care and Use of Laboratory Animals and were approved by the Institutional Animal Care and Use Committee of the University of Pittsburgh. MAM animals were treated following a previously established protocol (Moore et al, 2006). Briefly, timed pregnant female Sprague-Dawley rats (Harlan Research Models, Frederick, MD) were obtained at GD15 and housed individually in plastic breeding tubs. MAM (diluted in saline, $20 \mathrm{mg} / \mathrm{kg}$, i.p.) was administered on GD17. Matched control pregnant rats received injections of saline ( $1 \mathrm{ml} / \mathrm{kg}$, i.p.) on GD17. On day 21, offspring from 4 total litters (2 SAL and 2 MAM) were culled to 10 by removal of female pups to control for effects of the estrous cycle. Male pups were weaned on day 21 , housed on a reverse light cycle, and given free access to food and water in groups of two or three with littermates.

\section{Measuring Vocalizations and Freezing in Response to Footshock Stress}

Recording sessions were scheduled for postnatal day (PND) 22 (juvenile), 28 (weanling), 34 (mid adolescent), 37 (late adolescent), 42 (post-adolescent young adult), 50 (postadolescent young adult), and 54 (adult). Vocalizations were recorded from the same individuals at each of the above time points, SAL $N=12$, MAM $N=14$. Animals were selected in random order for 15-min recording sessions in a dimly-lit, soundproof operant box. The $16\left(\frac{1}{12}\right)^{\prime \prime} \times 16\left(\frac{1}{12}\right)^{\prime \prime} \times 15\left(\frac{1}{2}\right)^{\prime \prime}$ Plexiglass chamber was fitted with a grid floor comprised of $0.48 \mathrm{~cm}$ stainless steel rods spaced $1.6 \mathrm{~cm}$ apart and housed within a sound-attenuating cubicle (Med Associates, St Albans, VT). Each trial was initiated with a 60-s habituation period. Then five scrambled footshocks $(1.0 \mathrm{~mA}, 2 \mathrm{~s})$ were delivered at random intervals $(60 \pm 20 \mathrm{~s})$. Analog audio was acquired with a Pettersson D200 bat detector with display accuracy: $\pm 0.15 \mathrm{kHz}$ and bandwidth: $8 \pm 4 \mathrm{kHz}$ (Pettersson Elektronik, Carlisle, PA; Del Punta et al, 2002) tuned to $22 \mathrm{kHz}$ and digitized at $100 \mathrm{kHz}$ (AD Instruments, Colorado Springs, CO). All behavioral equipment was cleaned with ethanol between animals. Frequency-shifted signals were fed to speakers and behavior was monitored via a video camera. Vocalizations were counted and arranged in single-minute bins to measure rates. Intervals of $4 \mathrm{~s}$ were removed from the time period of shock delivery to eliminate noise associated with footshock-induced hyperlocomotion. Freezing, defined as an absence of all non-respiratory movement for at least $3 \mathrm{~s}$, was measured from videotape playback both during the footshock and during the entire test period.

\section{Measuring Plasma Corticosterone in Response to Footshock Stress}

MAM and SAL animals were exposed to one session of footshock per day for 10 days in the peripubertal period, from PND 31-40. The same individuals were exposed to footshock at each timepoint. In each session, rats were placed in a Plexiglas chamber equipped with a grid floor (see above). Twenty-five scrambled footshocks $(1.0 \mathrm{~mA}, 2 \mathrm{~s})$ were delivered every $60 \pm 20 \mathrm{~s}$ using Med-PC IV software (Med Associates, St Albans, VT). In the control condition (SHAM), rats were exposed to the same protocol but the grid floor was not connected to the current generator. All behavioral equipment was cleaned with ethanol between animals.

Blood was collected from the lateral tail vein before the first session of footshock (PND 31), immediately ( $<5 \mathrm{~min}$ ) after the second session (PND 32) and immediately after the last session (PND 40). Blood was collected in both groups in morning and afternoon time periods (counterbalanced) into EDTA-coated plastic tubes, immediately centrifuged, and stored at $-20^{\circ} \mathrm{C}$. An enzyme immunoassay was used to determine plasma concentrations of corticosterone (Assay Designs, Ann Arbor, MI). Corticosterone samples were diluted 1:40 and analyzed in duplicate.

\section{Statistics}

The effect of footshock on total number of calls, time spent vocalizing, rate of vocalization, and on freezing behavior was assessed using repeated measures two-way ANOVA. The effects of footshock on weight gain and plasma corticosterone were assessed using repeated measures two-way 
ANOVA and Tukey's post-hoc. All statistical analyses used $p>0.05$ and were presented as mean \pm SEM.

\section{RESULTS}

\section{Footshock-Evoked Vocalization}

Inescapable footshock stress reliably evoked $22 \mathrm{kHz}$ vocalizations in both SAL- and MAM-treated rats at each developmental time point. No animals were found to vocalize before the first footshock, ie, in the $60 \mathrm{~s}$ habituation period (see Methods). The signals were analyzed with regard to call duration and number of calls. Animals vocalized in short clusters or in continuous chains (Figure $1 \mathrm{~b}$ ). The frequency of $22 \mathrm{kHz}$ vocalizations occasionally shifted within the monitoring range of the detector. This modulation was most often present during the first call within a cluster and less evident in continuous vocalization chains, a pattern that is consistent with previous reports showing a similarly clustered $22 \mathrm{kHz}$ call frequency profile in rats responding to airpuff startle (Brudzynski and Holland, 2005).

Footshock provoked a broad range in the number of vocalizations across all animals within the 15-min trial period ( $210.0 \pm 13.7$, mean \pm SEM). Of the 26 rats tested, 18 did not respond in at least one session and only one animal did not respond at all over the course of the experiment. In any given session, an average of $6.4 \pm 0.8$ animals did not respond, but these animals did respond vigorously and often beyond the test duration in other sessions. No differences in treatment (MAM vs SAL) or litter were observed between responders and non-responders. Vocalizing over the entire $15 \mathrm{~min}$ range was less common
(21 occurrences): 13 animals vocalized over the entire test duration in at least one session, no more than 7 animals vocalized for over $15 \mathrm{~min}$ during a session, and there was only one instance in which an animal greater than 34 days old vocalized beyond the testing interval. The latency to first vocalization declined steadily from the first session $(190.9 \pm 15.2 \mathrm{~s})$ to the last $(76.8 \pm 12.8 \mathrm{~s})$. The duration of vocalization in MAM and SAL groups peaked in session 2 (weanling, 610.2 $\pm 52.2 \mathrm{~s}$; Figure 2a) and declined in session 4 (late adolescent, $240.6 \pm 36.42 \mathrm{~s}$ ), corresponding more closely to the length of the stimulus (five footshocks, interval $60 \pm 20 \mathrm{~s}$ ). Overall, clear group differences were observed across sessions, despite some degree of withinsession variability.

MAM Rats Demonstrated Higher Levels of Vocalization and More Freezing Behavior in Response to Repeated Footshock

In response to footshock, juvenile (PND 22) MAM rats elicited more $22 \mathrm{kHz}$ vocalizations throughout the 15 -min recording session than SAL controls (SAL $n=12$, MAM $n=14 ; \quad \mathrm{F}(1,23)=2.582, \quad p=0.119$, session $1: \quad t(2.868)$, $p=0.005$; Figure 1c). Although juvenile MAM animals did not vocalize more than their SAL counterparts during the period of intermittent, active footshock (ie, the first $5 \mathrm{~min}$ of each session; repeated measures two-way ANOVA, $t=1.447, p>0.05)$ they did emit a higher number of vocalizations following footshock ie, the last $10 \mathrm{~min}$ of each session (repeated measures two-way ANOVA, F $(1,23)=$ 4.049, $p<0.05$, significant effect in juvenile session, $t=2.312, p<0.05)$. This effect was not present at any other developmental timepoint.

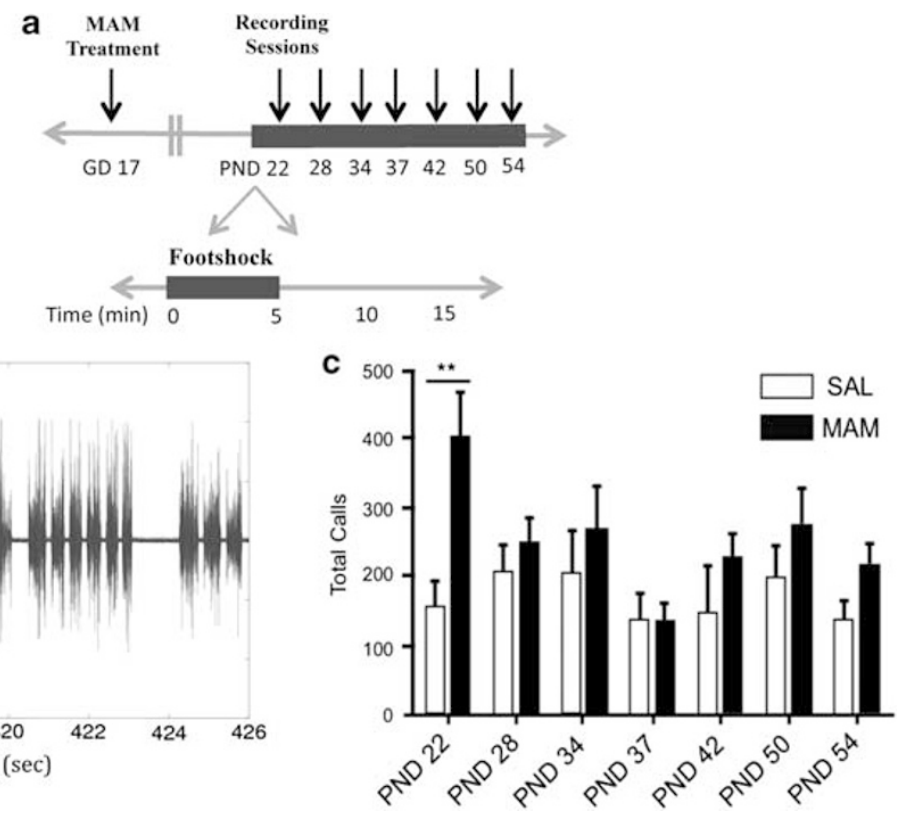

Figure I Juvenile MAM animals emitted more calls following footshock than SAL controls. (a) Design of USV experiment. MAM-treated animals tested at specific developmental time points were exposed to five footshocks delivered at random intervals (60 $\pm 20 \mathrm{~s}$ ) and $22 \mathrm{kHz}$ vocalizations were recorded throughout each I5-min session. Note that the same individuals were exposed to footshock at each developmental timepoint. (b) Sample trace illustrating the typical pattern of vocalization emitted by rats exposed to footshock stress. (c) MAM-treated animals in the juvenile period (PND 22) emitted more $22 \mathrm{kHz}$ vocalizations per I5-min session compared with SAL controls (*⿻丷木 $p<0.01, F(I, 23)=2.582, p=0.1$ I9, session I: $t(2.868), p=0.005)$. SAL $n=12$, $M A M n=14$. 

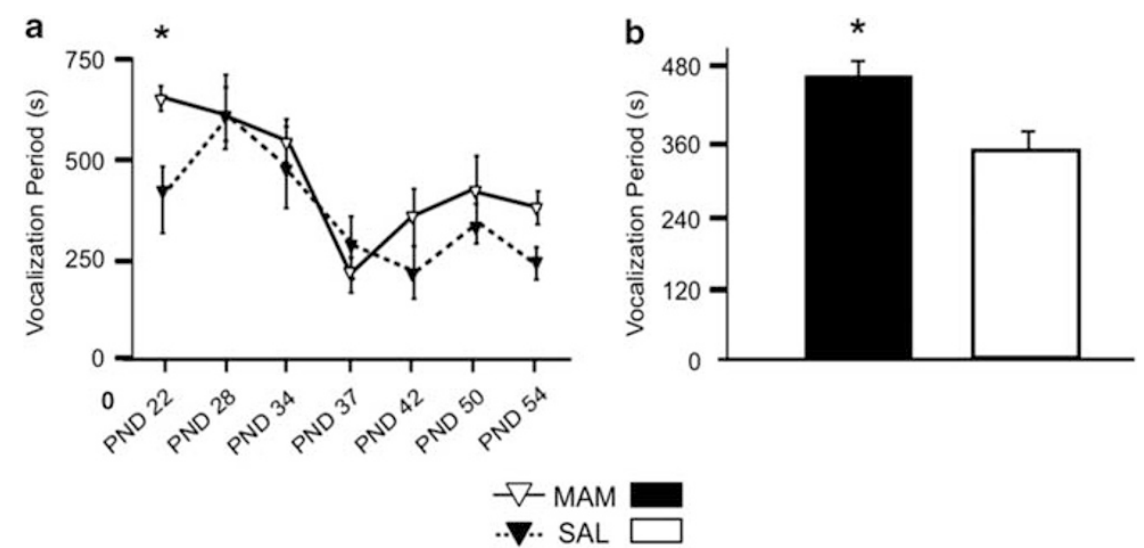

Figure 2 Juvenile MAM animals spent more time vocalizing following footshock than SAL controls. (a) MAM-treated animals in the juvenile period spent more time vocalizing compared to SAL controls, as measured from the beginning of the first vocalization to the end of the final vocalization in each I5-min session (*P $<0.05$, repeated measures two-way ANOVA, $F(I, 23)=7.569)$. (b) MAM animals spent more time vocalizing when measured across all ages tested (repeated measures two-way ANOVA, $F(I, 23)=7.569, p<0.01$ ) SAL $n=12$, MAM $n=\mid 4$.

In addition to producing more vocalizations, juvenile MAM animals also spent more time vocalizing than SAL controls, measured from the beginning of the first vocalization to the end of the final vocalization within each $15 \mathrm{~min}$ session. Furthermore, when measured across all developmental time points, MAM animals spent more time vocalizing than SAL controls overall (repeated measures two-way ANOVA, F(1, 23) = $7.569, p<0.01$; Figure $2 \mathrm{~b}$ ). There was no difference in the latency to first vocalization between MAM and SAL animals (repeated measures two-way ANOVA, $\mathrm{F}(1,23)=$ 2.657, $p>0.05$ ).

Juvenile MAM animals vocalized at a higher rate than SAL control animals following the period of active footshock, an effect not observed in older rats (repeated measures two-way ANOVA, $\mathrm{F}(1,23)=4.049, p>0.05$, session no. 1 : $t=2.312, p=0.023$; Figure 3 ). In addition, MAM animals vocalized at a higher rate than SAL controls during footshock across all sessions (repeated measures two-way ANOVA, $\mathrm{F}(1,23)=7.392, p<0.01)$. In particular, MAM rodents vocalized at a higher rate during the period of active footshock, ie, the first $5 \mathrm{~min}$ of each session, in the mid-adolescent phase (session 3: $t=2.143, p<0.05$ ) and during the young adult (session 5: $t=2.879, p<0.01$ ) phases.

Freezing behavior was measured both during the footshock, as well as across the entire 15-minute session. Both MAM and SAL rats exhibited similar levels of freezing during the footshock (repeated measures two-way ANOVA, $\mathrm{F}(1,24)=0.08, p=0.77)$. There was a significant increase in freezing during the footshock period in both groups across sessions (Figure 4a; repeated measures ANOVA, $F(6,24)=9.18, p<0.01)$. When measured across the entire $15 \mathrm{~min}$ session (both footshock and postfootshock), there were no group- or session-dependent differences between MAM and SAL rats (ANOVA, $\mathrm{F}(1,24)=0.13, p=0.72)$. However, MAM rats at PND22 showed significantly more freezing, owing to increased post-shock freezing compared with controls (Figure $4 \mathrm{~b}$; $t(2.2137), p=0.04)$.

\section{Physiological Responses to Repeated Footshock were Blunted in MAM Animals}

Statistical analysis of weight gain in MAM and SAL animals over the course of footshock treatment revealed a significant effect of time (SAL-SHAM $n=7$, SAL-FS $n=7$, MAM-SHAM $n=5$, MAM-FS $n=12$; repeated measures two-way ANOVA, $\mathrm{F}(9351)=1092, p<0.01$; Figure $5 \mathrm{~b})$ and treatment $(\mathrm{F}(3,39)=11.25, p<0.01)$ as well as a significant interaction between time and treatment $(\mathrm{F}(27351)=7.629$, $p<0.01)$. SAL controls exposed to repeated footshock in the peripubertal period gained significantly less weight over the course of treatment than SAL animals exposed to SHAM (Tukey's post-hoc, $p<0.05$ ). In contrast, MAM animals exposed to repeated footshock gained a similar amount of weight over the course of treatment as MAM animals exposed to SHAM (Tukey's post-hoc, p>0.05, Figure 5b).

Measurement of plasma corticosterone levels revealed divergent responses to SHAM exposure in SAL and MAM animals. Before the first treatment session, corticosterone levels in all groups were low $(\sim 13.35 \mathrm{ng} / \mathrm{ml}$ on average), consistent with a basal level of HPA axis activation (Figure 5; Lightman et al, 2008). In SAL animals exposed to SHAM treatment, there was an overall significant effect of day (repeated measures one-way ANOVA, $\mathrm{F}(2,6)=19.33$, $p<0.01)$. Following the second session of SHAM exposure, corticosterone levels in SAL animals were significantly elevated $(77 \pm 11 \mathrm{ng} / \mathrm{ml}$; Tukey's post-hoc, $p<0.01)$. However, plasma corticosterone levels measured after the tenth day of SHAM exposure had returned to near-baseline levels, (11.5 $\pm 1.1 \mathrm{ng} / \mathrm{ml}$; Figure $5 \mathrm{c})$, suggesting adaptation of the HPA axis response to SHAM exposure/blood draw. In contrast to their SAL counterparts, following the second session of SHAM exposure corticosterone levels in MAM animals remained unchanged (repeated measures one-way ANOVA $\mathrm{F}(2,8)=2.351, p>0.05,95 \% \mathrm{CI}=-18.22$ to 62.78$)$. These levels remained unchanged even after the tenth session of SHAM exposure $(43.0 \pm 19 \mathrm{ng} / \mathrm{ml}$; Figure $5 \mathrm{c}$ ), suggesting a lack of initiation and adaptation 


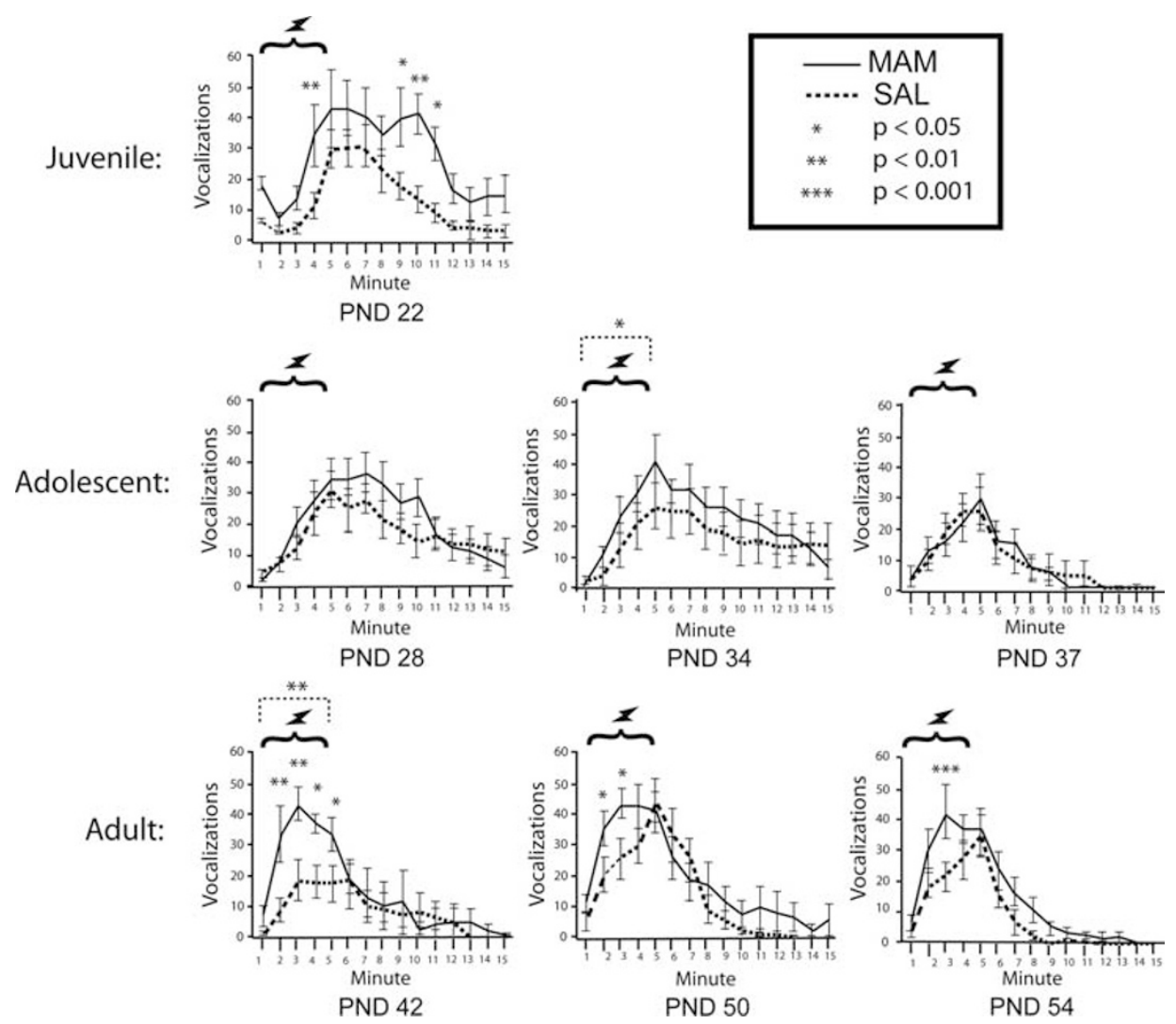

Figure 3 Juvenile MAM animals vocalized at a higher rate following footshock compared with SAL controls. Juvenile MAM animals emitted $22 \mathrm{kHz}$ vocalizations at a significantly higher rate following the period of active footshock as compared with SAL controls, an effect not observed in other stages of development (repeated measures two-way ANOVA, $F(I, 23)=4.049, p<0.05, t=2.312, p<0.05$ ). Across all sessions, MAM-treated animals vocalized at a higher rate compared with SAL controls during the period of active footshock ie, the first 5 min of each session (repeated measures two-way ANOVA, $F(I, 23)=7.392, p<0.05)$, with significant interactions in the mid-adolescent (PND 34) and young adult (PND 42) stages (PND 34: $t=2.143, p<0.05$; PND 42: $t=2.879, p<0.01)$.
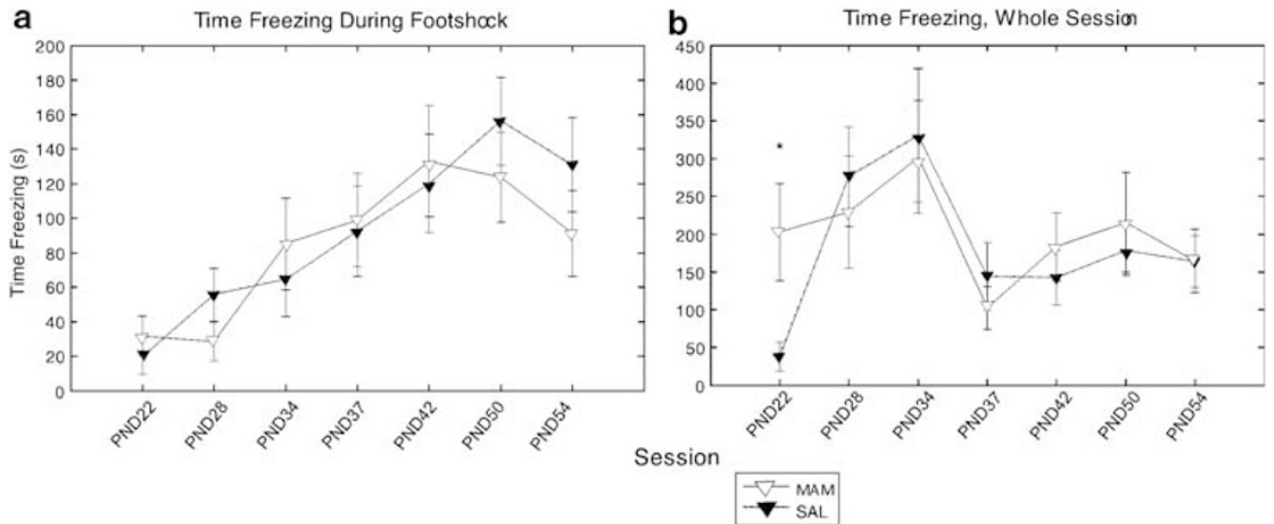

Figure 4 MAM animals showed increased freezing during post-footshock periods. Freezing was measured during the footshock, as well as across the entire 15 min session. (a) When measured only during the footshock, both MAM-treated rats and SAL-treated rats showed increased levels of freezing across sessions. No differences between MAM and SAL animals were observed. (b) When measured across the entire 15 min period, there were no differences overall between MAM and SAL rats; however, the MAM rats sshowed significantly greater time freezing at PND22, which is consistent with the increased vocalizations observed beyond the period of footshock exposure.

of corticosterone responses to SHAM exposure in MAM animals.

Stress responses in SAL and MAM animals exposed to footshock treatment mirrored the SHAM pattern but were much greater in amplitude. In SAL animals exposed to footshock, there was an overall significant effect of day (repeated measures one-way ANOVA, $\mathrm{F}(2,5)=21.43$, $p<0.01)$. SAL animals exposed to footshock displayed an increase in plasma corticosterone levels following the second session of footshock $(421 \pm 67 \mathrm{ng} / \mathrm{ml})$; a nearly 

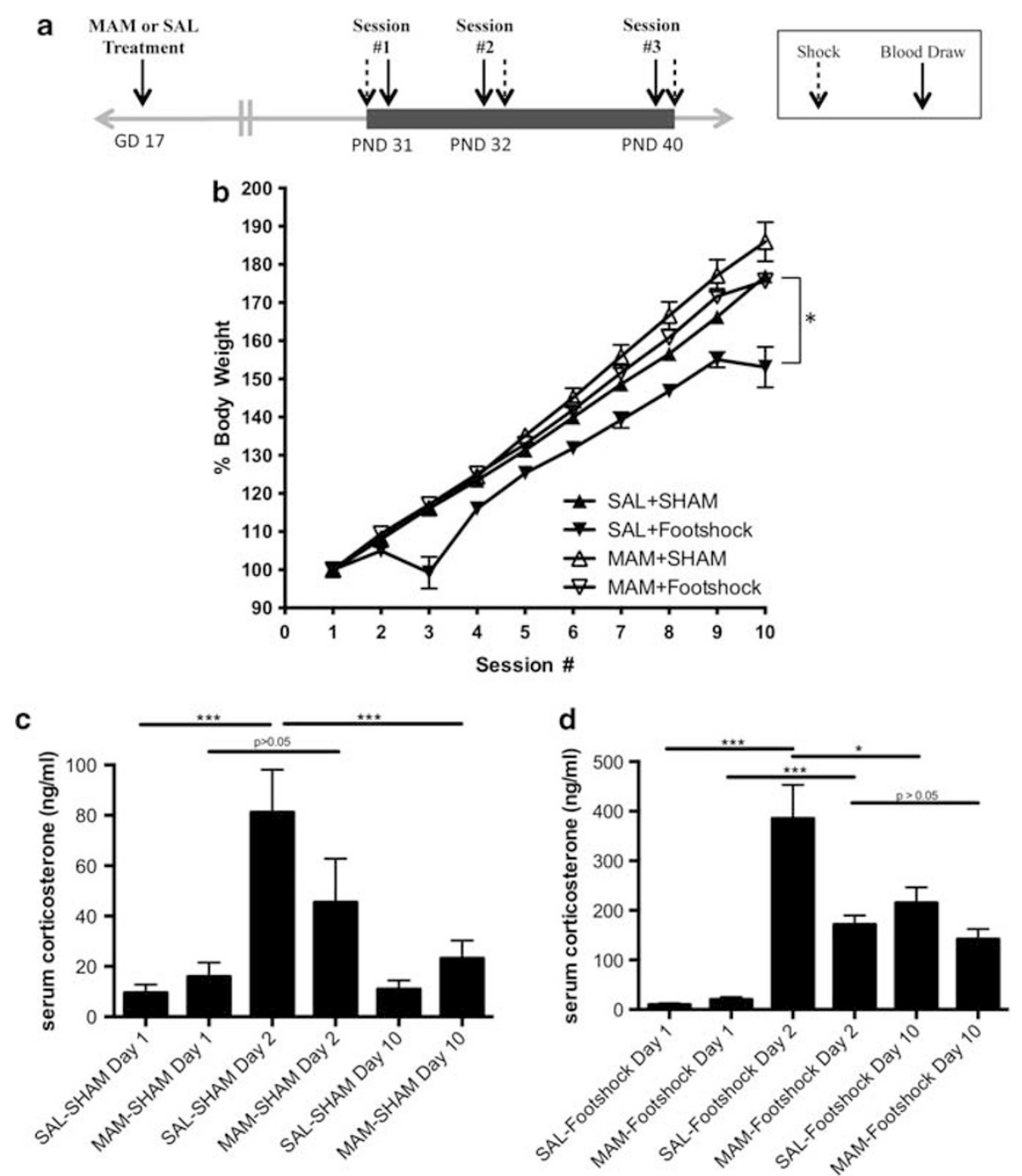

Figure 5 MAM animals displayed abnormal weight gain and HPA axis responses to acute and repeated footshock exposure. (a) Design of corticosterone experiment. A separate cohort of MAM-treated animals were exposed to 10 days of repeated footshock stress (dashed arrow) in the peripubertal period (PND 3 -40) and plasma corticosterone levels were measured in blood drawn (solid arrows) before the first session (PND 31 ), immediately after the second session (PND 32) and immediately after the last session (PND 40) in the same animals, as indicated by the position of the arrows. (b) SAL-treated animals gained significantly less weight in response to repeated footshock as compared with SHAM controls (repeated measures two-way ANOVA, Tukey's post-hoc, $\left.{ }^{*} p<0.05\right)$. In contrast, MAM animals exposed to repeated footshock gained a similar amount of weight compared with their SAL counterparts (repeated measures two-way ANOVA, Tukey's post-hoc, p > 0.05). (c) SAL animals exposed to the SHAM condition displayed a moderate but significant increase in plasma corticosterone levels following the second session that adapted over the course of I0 sessions (repeated measures one way ANOVA $F(2,6)=19.33, p<0.01$, Tukey's post-hoc). (Note differences in y axis between SHAM and footshock treatments.) MAM animals exposed to the SHAM condition showed no differences in plasma corticosterone levels following the second or tenth session (repeated measures one-way ANOVA $F(2,8)=2.35 I$, p $>0.05$, Tukey's post-hoc). (d) Corticosterone levels in SAL animals were elevated ( $\sim 40$-fold increase) after the second session (repeated measures one-way ANOVA F $(2,5)=21.43$, Tukey's post-hoc **** $p<0.00 \mathrm{I}$ ) and had adapted by the tenth session (repeated measures one-way ANOVA $F(2,5)=21.43$. Tukey's post-hoc $* p<0.05)$. In contrast, MAM animals exposed to footshock displayed a significant, but attenuated increase in plasma corticosterone ( $\sim$-fold increase) following the second session of treatment (repeated measures one-way ANOVA F(2,II) $=24.34$, Tukey's post-hoc, $* p<0.05$ ) that did not adapt by the tenth session (repeated measures one-way ANOVA F $(2,1 \mathrm{I})=24.34$, Tukey's post-hoc, p $>0.05$ ). For all, SAL-SHAM $n=7$, SAL-FS $n=7$, MAM-SHAM $n=5$, MAM-FS $n=12$.

200-fold increase over baseline levels (Tukey's post-hoc, $p<0.01$; Figure 5d). After the tenth session of footshock, this response was significantly reduced $(249 \pm 21 \mathrm{ng} / \mathrm{ml}$; Tukey's post-hoc, $p<0.05$ ), consistent with an adaptation of the corticosterone response to stress in SAL animals. In MAM animals exposed to footshock treatment, there was a significant effect of day on corticosterone responses (repeated measures one-way ANOVA $\mathrm{F}(2,11)=24.34$, $p<0.01)$. In contrast to SAL animals, however, plasma corticosterone levels following footshock exposure were blunted in MAM animals. After the second session of footshock, corticosterone levels were increased 8-fold over baseline levels (Tukey's post-hoc, $p<0.05,163.0 \pm 15 \mathrm{ng} / \mathrm{ml}$; Figure 5d), a much less robust response than observed in SAL animals. In addition, this response did not adapt over time. Corticosterone measured in MAM animals after the tenth session remained elevated near levels observed following the second session $(130.0 \pm 17 \mathrm{ng} / \mathrm{ml})$ even after 10 days of footshock exposure (Tukey's post-hoc, $p>0.05$ ). This is in contrast to the pronounced decrease in plasma 
corticosterone observed in SAL animals following the tenth session of footshock.

\section{DISCUSSION}

Our measurements of USVs emitted in response to acute footshock stress show that MAM rats in the juvenile period are more responsive to stressors as compared with matched SAL rats, at least as represented by our footshock stress paradigm. In response to acute footshock, juvenile MAMtreated rats emitted more $22 \mathrm{kHz}$ vocalizations than their SAL counterparts, vocalized for a longer duration, and emitted vocalizations more frequently compared with SAL controls. In the same experimental protocol, both MAM and SAL rats showed equivalent amounts of freezing during the footshock, but juvenile MAM rats exhibited more nonshock freezing. The fact that both groups showed similar freezing during the footshock suggests that MAM and SAL rats are equally sensitive to the footshock. Moreover, adolescent MAM rats showed a disruption in HPA axis responses to stress, exhibiting attenuated corticosterone levels after acute footshock that, unlike in SAL counterparts, did not change after 10 days of stress exposure. Adolescent MAM rats also did not show diminished weight gain due to footshock exposure, unlike their SAL counterparts. Although we have not observed evidence to date of differences in litters of MAM rats, it should be noted that 21 of SAL and 21 of MAM animals were used for our experiments, as described in the Methods. Taken together, the USV findings suggest that juvenile and adolescent MAM animals are more sensitive to the effects of stress, while the weight gain and corticosterone data suggest that they are unable to integrate appropriate responses to acute stress and unable to adapt to repeated stress over time.

Previous studies have demonstrated that $22 \mathrm{kHz}$ USVs and HPA axis responses to stress change with developmental disruption. Kosten et al (2005) demonstrated that rats exposed to neonatal isolation show increased USV responses to footshock stress. In addition, rats exposed to neonatal ventral hippocampal lesion display an attenuated increase in plasma corticosterone levels as compared with sham-lesion controls after 20 min of footshock, and this response fails to adapt after $60 \mathrm{~min}$ of footshock (Chrapusta et al, 2003). Similarly, the offspring of dams exposed to chronic unpredictable stress during pregnancy display an exaggerated corticosterone response to an open field test (Clinton et al, 2008). Taken together, these data suggest that USV and HPA axis responses to stress are sensitive to various developmental disruptions and that HPA axis dysregulation could contribute to other abnormal phenotypes seen in animal models of psychiatric disease.

In addition to our data in juvenile MAM animals, we also observed an increase in USV frequency in adult MAM animals in response to footshock. Although these data are difficult to interpret given the relative lack of previous work concerning USV emission across the rat lifespan, we believe that this effect is independent of the effect we observed in juvenile animals. Although call frequency was significantly elevated in this group, call number and duration were not. In addition, the increases in USV frequency and freezing occurred during the period of active footshock, in contrast to juvenile animals that showed heightened vocalizations and freezing following the footshock. Finally, the effect appears to be unrelated to any sort of learning response, as no similar effect was observed in the adolescent or young adult time points. Instead, this may reflect a continuation of increased stress reactivity in the adult, albeit at a lower intensity.

MAM animals exposed to footshock stress for 10 consecutive days did not show any differences in weight gain as compared with their SHAM counterparts. In contrast, SAL animals exposed to the same footshock paradigm did in fact show a significant reduction in weight gain over the 10-day paradigm, as is typically observed in chronic stress protocols (Dess and Minor, 1989; DiasFerreira et al, 2009; Jankord and Herman, 2008). Indeed, the effects of stress on the HPA axis and the subsequent endocrine response are known to influence appetite as well as metabolic processes (McEwen, 2007). We interpret this unexpected lack of response in adolescent MAM animals to the stress paradigm as an aberrant metabolic response to stress that could be related to the HPA axis abnormalities we have identified.

Although the same animals were exposed to stressors at each developmental time point in our USV experiment, we do not believe that this impacted the differences we observed between the MAM and SAL groups for several reasons. Exposures were very brief and separated by several days. In addition, adult MAM animals were found to vocalize at a higher frequency in response to footshock. This finding suggests against the occurrence of a learning response and/or habituation, and even more so given that it was observed during the period of active footshock, in contrast to juvenile animals who vocalized at a higher rate following active footshock. Finally, if repeated stress exposure on its own had an effect on USV number, duration, or frequency, we would expect that such an effect would be observed in both the SAL and MAM groups. However, this was not the case: while SAL animals responded in a similar pattern across the lifespan, MAM animals displayed a characteristic increase in call number, duration, and frequency at PND 22.

Perhaps most convincingly, we observed no differences in freezing behavior during the footshock across all treatment groups and sessions, although both MAM and SAL rats showed increased time freezing during the footshock across developmental periods. Although the difference in USVs in the youngest cohort is robust, it is difficult to evaluate whether the effects on vocalization seen in the adult may have been due to differences in learning with repeated exposure between MAM and SAL rats, or were due solely to the MAM treatment. Nonetheless, the differences in MAM rats at the juvenile stage at first footshock exposure and the lack of an apparent difference in learning response between SAL and MAM animals suggests that any differences we observed in USVs in MAM animals were likely due to the MAM treatment itself.

Interestingly, the abnormal response to stress identified in our work is present pre- and peripubertally; a time that precedes the point of transition to psychosis in susceptible individuals (Addington and Heinssen, 2012; Miller et al, 2001) and also precedes the time point that MAM rats show increased amphetamine-induced locomotor responses 
(Flagstad et al, 2004; Moore et al, 2006). The finding of a blunted corticosterone response to acute footshock exposure is of particular interest. Studies have shown that a substantial corticosterone response is necessary for homeostatic adaptation to stressors (McEwen and Gianaros, 2010; Rao et al, 2012), and a pathologically blunted cortisol response to stress in humans is believed to contribute to disorders such as post-traumatic stress disorder (Baker et al, 2005; LaBar and Cabeza, 2006; Luo et al, 2012). Therefore, a blunted initial corticosterone response and a failure to show changes with repeated exposure could contribute to the pathological responses to stress seen in the MAM model.

In summary, in pre- and peripubertal periods there is an increase in USVs in response to stress as well as disruption of the corticosterone response in MAM rats. However, the source of this increased responsivity is unclear. Although this study does not directly address cellular and molecular mechanisms of this phenomenon, one possibility is that it could result from a failure of higher cortical processes to limit the impact of stressors. The prefrontal cortex is a region that has been linked to the pathophysiology of schizophrenia (Knable and Weinberger, 1997; Lewis et al, 2005), and it has been suggested that deficits in this region could be present in the prodromal state of schizophrenia (Phillips and Seidman, 2008; Thompson et al, 2004). Moreover, the mPFC has been shown to limit the response to stress in rats and in humans (Finlay et al, 1995; Hariri et al, 2003). Therefore, a failure of the mPFC to regulate stress in the MAM-treated rat or in the prodromal stage of schizophrenia could contribute to pathological changes in the hippocampus observed in this disorder. It has been shown that activation of the amygdala, which occurs during stress, will lead to parvalbumin interneuron loss in the hippocampus (Berretta et al, 2001) and loss of hippocampal parvalbumin interneurons is proposed to lead to the DA hyper-responsivity in the MAM model (Lodge and Grace, 2007). Thus, a hypersensitivity to stress potentially secondary to decreased mPFC function could initiate a cascade of events during juvenile/adolescent stages to lead to hippocampal dysfunction and the emergence of the schizophrenia-like phenotype observed in the MAM model (Grace, 2004; Thompson et al, 2004).

These findings, when considered in light of work demonstrating hippocampal dysfunction in MAM animals, suggest a mechanism for schizophrenia pathogenesis whereby the deleterious effects of stress on the hippocampus are driven by a failure of the MPFC to regulate stress. The resulting profound hippocampal dysfunction would in turn prevent an individual from mounting appropriate homeostatic responses to stressors, rendering them more vulnerable to the effects of stress, and increasing their risk for developing schizophrenia. This is also consistent with human epidemiological data showing that, in children at risk for schizophrenia, those that show the greatest response to stressors tend to convert (Johnstone et al, 2002; Owens et al, 2005). If stress during puberty and adolescence is indeed a contributing factor to the transition to psychosis, controlling stress at this vulnerable period may circumvent these pathological changes and prevent the emergence of psychosis later in life (Du and Grace, 2013; Thompson et al, 2004).

\section{FUNDING AND DISCLOSURE}

AAG has received compensation from Johnson and Johnson, Lundbeck, Pfizer, GSK, Puretech Ventures, Merck, Takeda, Dainippon Sumitomo, Otsuka, Lilly, Roche, and Asubio over the past 3 years. ECZ, MB, and SGE declare no conflict of interest.

\section{ACKNOWLEDGEMENTS}

This work was funded by NIH MH57440. The authors would like to thank all members of the Grace lab for their helpful input and advice.

\section{REFERENCES}

Addington J, Heinssen R (2012). Prediction and prevention of psychosis in youth at clinical high risk. Annu Rev Clin Psychol 8: 269-289.

Baker DG, Ekhator NN, Kasckow JW, Dashevsky B, Horn PS, Bednarik L et al (2005). Higher levels of basal serial CSF cortisol in combat veterans with posttraumatic stress disorder. Am J Psychiatry 162: 992-994.

Belujon P, Grace AA (2011). Hippocampus, amygdala, and stress: interacting systems that affect susceptibility to addiction. Ann N Y Acad Sci 1216: 114-121.

Belujon P, Patton MH, Grace AA (2013). Role of the prefrontal cortex in altered hippocampal-accumbens synaptic plasticity in a developmental animal model of schizophrenia. Cerebral Cortex (doi:10.1093/cercor/bhs380).

Benes F (1997). The role of stress and dopamine-GABA interactions in the vulnerability for schizophrenia. J Psychiatr Res 31: 257-275.

Berretta S, Munno DW, Benes FM (2001). Amygdalar activation alters the hippocampal GABA system: "partial" modelling for postmortem changes in schizophrenia. J Comp Neurol 431: 129-138.

Brudzynski SM (2001). Pharmacological and behavioral characteristics of $22 \mathrm{kHz}$ alarm calls in rats. Neurosci Biobehav Rev 25: 611-617.

Brudzynski SM, Holland G (2005). Acoustic characteristics of air puff-induced $22-\mathrm{kHz}$ alarm calls in direct recordings. Neurosci Biobehav Rev 29: 1169-1180.

Chance SA, Esiri MM, Crow TJ (2002). Amygdala volume in schizophrenia: post-mortem study and review of magnetic resonance imaging findings. Br J Psychiatry 180: 331-338.

Chrapusta SJ, Egan MF, Wyatt RJ, Weinberger DR, Lipska BK (2003). Neonatal ventral hippocampal damage modifies serum corticosterone and dopamine release responses to acute footshock in adult Sprague-Dawley rats. Synapse 47: 270-277.

Clinton S, Miller S, Watson SJ, Akil H (2008). Prenatal stress does not alter innate novelty-seeking behavioral traits, but differentially affects individual differences in neuroendocrine stress responsivity. Psychoneuroendocrinology 33: 162-177.

Corcoran C, Walker E, Huot R, Mittal V, Tessner K, Kestler L et al (2003). The stress cascade and schizophrenia: etiology and onset. Schizophr Bull 29: 671-692.

Del Punta K, Leinders-Zufall T, Rodriguez I, Jukam D, Wysocki CJ, Ogawa $S$ et al (2002). Deficient pheromone responses in mice lacking a cluster of vomeronasal receptor genes. Nature 419: 70-74.

Dess N, Minor T (1989). Suppression of feeding and body weight by inescapable shock: modulation by quinine adulteration, stress reinstatement, and controllability. Physiol Behav 45: 975-983.

Dias-Ferreira E, Sousa J, Melo I, Morgado P (2009). Chronic stress causes frontostriatal reorganization and affects decision-making. Science 325: 621-625. 
Drugan RC, Christianson JP, Stine WW, Soucy DP (2009). Swim stress-induced ultrasonic vocalizations forecast resilience in rats. Behav Brain Res 202: 142-145.

$\mathrm{Du}$ Y, Grace AA (2013). Peripubertal diazepam prevents the emergence of dopamine hyper-responsivity in the MAM model of schizophrenia. Neuropsychopharmacology (doi:10.1038/npp. 2013.101)

Finlay JM, Zigmond MJ, Abercrombie ED (1995). Increased dopamine and norepinephrine release in medial prefrontal cortex induced by acute and chronic stress: effects of diazepam. Neuroscience 64: 619-628.

Flagstad P, Mørk A, Glenthøj BY, van Beek J, Michael-Titus AT, Didriksen M (2004). Disruption of neurogenesis on gestational day 17 in the rat causes behavioral changes relevant to positive and negative schizophrenia symptoms and alters amphetamineinduced dopamine release in nucleus accumbens. Neuropsychopharmacology 29: 2052-2064.

Goto Y, Grace AA (2006). Alterations in medial prefrontal cortical activity and plasticity in rats with disruption of cortical development. Biol Psychiatry 60: 1259-1267.

Grace AA (2004). Developmental dysregulation of the dopamine system and the pathophysiology of schizophrenia. In: Keshavan M, Kennedy JL, Murray RM (eds). Neurodevelopment and Schizophrenia. Cambridge University Press, pp 273-294.

Grace AA (2010). Dopamine system dysregulation by the ventral subiculum as the common pathophysiological basis for schizophrenia psychosis, psychostimulant abuse, and stress. Neurotox Res 18: 367-376.

Grace AA, Moore H (1998). Regulation of information flow in the nucleus accumbens: A model for the pathophysiology of schizophrenia. American Psychological Association: Washington, DC, USA, pp 123-157.

Hariri AR, Mattay VS, Tessitore A, Fera F, Weinberger DR (2003). Neocortical modulation of the amygdala response to fearful stimuli. Biol Psychiatr 53: 494-501.

Jankord R, Herman JP (2008). Limbic regulation of hypothalamopituitary-adrenocortical function during acute and chronic stress. Ann N Y Acad Sci 1148: 64-73.

Johnstone EC, Lawrie SM, Cosway R (2002). What does the Edinburgh high-risk study tell us about schizophrenia? Am J Med Genet 114: 906-912.

Kim EJ, Kim ES, Covey E, Kim JJ (2010). Social transmission of fear in rats: the role of $22-\mathrm{kHz}$ ultrasonic distress vocalization. PLOS ONE 5: e15077.

Knable MB, Weinberger DR (1997). Dopamine, the prefrontal cortex and schizophrenia. J Psychopharmacol 11: 123-131.

Kosten TA, Miserendino MJD, Bombace JC, Lee HJ, Kim JJ (2005). Sex-selective effects of neonatal isolation on fear conditioning and foot shock sensitivity. Behav Brain Res 157: 235-244.

LaBar KS, Cabeza R (2006). Cognitive neuroscience of emotional memory. Nat Rev Neurosci 7: 54-64.

Le Pen G, Gourevitch R, Hazane F, Hoareau C, Jay TM, Krebs M-O (2006). Peri-pubertal maturation after developmental disturbance: a model for psychosis onset in the rat. Neuroscience 143: 395-405.

Lewis DA, Hashimoto T, Volk DW (2005). Cortical inhibitory neurons and schizophrenia. Nat Rev Neurosci 6: 312-324.

Lightman SL, Wiles CC, Atkinson HC, Henley DE, Russell GM, Leendertz JA et al (2008). The significance of glucocorticoid pulsatility. Eur J Pharmacol 583: 255-262.

Lim C, Chong S (2009). Psychosocial factors in the neurobiology of schizophrenia: a selective review. Ann Acad Med Singapore 38: 402-406.

Lisman JE, Pi HJ, Zhang Y, Otmakhova NA (2010). A thalamohippocampal-ventral tegmental area loop may produce the positive feedback that underlies the psychotic break in schizophrenia. Biol Psychiatr 68: 17-24.

Lodge DJ, Grace AA (2007). Aberrant hippocampal activity underlies the dopamine dysregulation in an animal model of schizophrenia. J Neurosci 27: 11424-11430.

Lodge DJ, Grace AA (2008). Hippocampal dysfunction and disruption of dopamine system regulation in an animal model of schizophrenia. Neurotox Res 14: 97-104.

Luo H, Hu X, Liu X, Ma X, Guo W, Qiu C et al (2012). Hair cortisol level as a biomarker for altered hypothalamic-pituitary-adrenal activity in female adolescents with posttraumatic stress disorder after the 2008 Wenchuan earthquake. Biol Psychiatr 72: 65-69.

McEwen BS (2007). Physiology and neurobiology of stress and adaptation: central role of the brain. Physiol Rev 87: 873-904.

McEwen BS, Gianaros PJ (2010). Central role of the brain in stress and adaptation: links to socioeconomic status, health, and disease. Ann N Y Acad Sci 1186: 190-222.

Meyer-Lindenberg A, Tost H (2012). Neural mechanisms of social risk for psychiatric disorders. Nat Neurosci 15: 663-668.

Miller P, Lawrie SM, Hodges A, Clafferty R, Cosway R, Johnstone EC (2001). Genetic liability, illicit drug use, life stress and psychotic symptoms: preliminary findings from the Edinburgh study of people at high risk for schizophrenia. Soc Psychiatr Psychiatr Epidemiol 36: 338-342.

Moore H, Jentsch JD, Ghajarnia M, Geyer MA, Grace AA (2006). A neurobehavioral systems analysis of adult rats exposed to methylazoxymethanol acetate on E17: implications for the neuropathology of schizophrenia. Biol Psychiatry 60: 253-264.

Nagata Y, Matsumoto H (1969). Studies on methylazoxymethanol: methylation of nucleic acids in the fetal rat brain. Proc Soc Exp Biol Med 132: 383.

Owens DGC, Miller P, Lawrie SM, Johnstone EC (2005). Pathogenesis of schizophrenia: a psychopathological perspective. Br J Psychiatr 186: 386-393.

Phillips LK, Seidman LJ (2008). Emotion processing in persons at risk for schizophrenia. Schizophr Bull 34: 888-903.

Portfors CV (2007). Types and functions of ultrasonic vocalizations in laboratory rats and mice. J Am Assoc Lab Anim Sci 46: $28-34$.

Rao RP, Anilkumar S, McEwen BS, Chattarji S (2012). Glucocorticoids protect against the delayed behavioral and cellular effects of acute stress on the amygdala. Biol Psychiatr 72: 466-475.

Roozendaal B, McEwen BS, Chattarji S (2009). Stress, memory and the amygdala. Nat Rev Neurosci 10: 423-433.

Schwarting RKW, Wöhr M (2012). On the relationships between ultrasonic calling and anxiety-related behavior in rats. Braz J Med Biol Res 45: 337-348.

Thompson JL, Pogue-Geile MF, Grace AA (2004). Developmental pathology, dopamine, and stress: a model for the age of onset of schizophrenia symptoms. Schizophr Bull 30: 875-900.

Touma C (2011). Stress and affective disorders: animal models elucidating the molecular basis of neuroendocrine-behavior interactions. Pharmacopsychiatry 44: S15-S26.

Tsuang M (2000). Schizophrenia: genes and environment. Biol Psychiatr 47: 210-220.

Walker E, Mittal V, Tessner K (2008). Stress and the hypothalamic pituitary adrenal axis in the developmental course of schizophrenia. Annu Rev Clin Psychol 4: 189-216.

Walker EF, Sabuwalla Z, Huot R (2004). Pubertal neuromaturation, stress sensitivity, and psychopathology. Dev Psychopathol 16: 807-824.

Williams AM, Reis DJ, Powell AS, Neira LJ, Nealey KA, Ziegler CE et al (2012). The effect of intermittent alcohol vapor or pulsatile heroin on somatic and negative affective indices during spontaneous withdrawal in Wistar rats. Psychopharmacology 223: 75-88. 